\title{
2010 Engineering Salaries: AAES Tallies Industry Survey Results
}

\author{
Maureen Byko
}

What's an engineer worth these days? The easy answer: It depends. How long did this engineer go to school? Is he/she a supervisor? Can we specify a particular engineering discipline? The combination of variables could be endless, but in the annual engineering salary survey conducted by the Engineering Workforce Commission (EWC) of the American Association of Engineering Societies (AAES), the focus is limited to certain key factors: degree and supervisory status, engineering discipline, years in the profession, geographic location, and size of employer. To sum up the report, an engineer's best bet for the highest salary is to have $35+$ years experience and be a supervisor with a Ph.D. working in a small company in the Chicago Metropolitan area.

In this excerpt from the most recent EWC report, Engineers' Salaries: Special Industry Report 2010, Table I shows median salaries by discipline; Table II, salaries based on experience, supervisory responsibility, and level of education; and Table III, salaries based on experience and employer.

The EWC has been gathering information about engineering compensation since 1953. The data is gleaned from employers that agree to participate in the survey, with a goal of providing estimates of the most current salaries for all employed engineers. This summary provides some highlights of the 2010 report which reflects base salaries effective February 1, 2010. Employers from industry and government participated, representing 35,938 engineers. For the full report or to participate in the 2011 salary survey, visit the AAES website at AAES.org.

\section{Maureen Byko is editor of JOM.}

\begin{tabular}{lccccccc}
\hline \multicolumn{7}{c}{ Table I. Median Annual Salaries for Engineers by Discipline (in Dollars) } \\
\hline & \multicolumn{7}{c}{ Number of Years after B.S. } \\
\cline { 2 - 7 } Discipline & $\mathbf{0}$ & $\mathbf{5}$ & $\mathbf{9 - 1 0}$ & $\mathbf{1 3 - 1 6}$ & $\mathbf{2 1 - 2 5}$ & $\mathbf{3 5 +}$ \\
\hline Aerospace Engineers & - & - & & 85,888 & 98,995 & 113,964 & 122,618 \\
Computer Engineers & - & 82,998 & 95,284 & 104,767 & 122,364 & 120,332 \\
Chemical Engineers & - & 68,399 & 82,570 & 88,049 & 101,394 & 114,372 \\
Civil Engineers & 54,358 & 62,899 & 70,126 & 80,159 & 88,522 & 96,884 \\
Electrical Engineers & - & 72,787 & 83,626 & 93,909 & 106,243 & 106,725 \\
Environmental Engineers & - & - & 67,740 & 83,300 & 100,791 & 104,346 \\
General Engineers & - & 78,373 & 94,417 & 99,090 & 106,039 & 107,879 \\
Industrial Engineers & - & 73,094 & 85,571 & 91,517 & 105,562 & 105,510 \\
Materials Engineers & - & - & 83,369 & 96,392 & 114,658 & 129,635 \\
Mechanical Engineers & 57,790 & 73,414 & 84,965 & 95,587 & 108,099 & 119,967 \\
Nuclear Engineers & - & - & - & 92,833 & 99,817 & 121,900 \\
\hline
\end{tabular}

Table II. Median Annual Salaries for Engineers Based on Experience, Supervisory Responsibility, and Level of Education (in Dollars)

\begin{tabular}{|c|c|c|c|c|c|c|}
\hline \multirow[b]{2}{*}{ Employer } & \multicolumn{6}{|c|}{ Number of Years after B.S. } \\
\hline & 0 & 5 & $9-10$ & $13-16$ & $21-25$ & $35+$ \\
\hline \multicolumn{7}{|c|}{$\overline{\text { Nonsupervisory }}$} \\
\hline B.S. & 60,898 & 71,374 & 78,620 & 84,901 & 93,899 & 95,561 \\
\hline M.S. & - & 82,616 & 91,789 & 96,922 & 101,838 & 103,519 \\
\hline Ph.D. & - & - & 86,791 & 90,399 & 97,243 & 116,464 \\
\hline \multicolumn{7}{|l|}{ Supervisory } \\
\hline B.S. & - & - & 86,223 & 92,902 & 106,111 & 116,749 \\
\hline M.S. & - & 114,221 & 115,969 & 121,844 & 129,071 & 114,150 \\
\hline Ph.D. & - & - & - & 119,680 & 133,424 & 152,961 \\
\hline
\end{tabular}

Table III. Median Annual Salaries for Engineers Based on Experience and Employer (in Dollars)

\begin{tabular}{|c|c|c|c|c|c|c|}
\hline \multirow[b]{2}{*}{ Employer } & \multicolumn{6}{|c|}{ Number of Years after B.S. } \\
\hline & 0 & 5 & $9-10$ & $13-16$ & $21-25$ & $35+$ \\
\hline \multicolumn{7}{|l|}{$\overline{\text { Architecture, Engineering, and }}$} \\
\hline Related Services & 57,562 & 71,076 & 85,061 & 97,644 & 103,914 & 105,564 \\
\hline Primary Metal Manufacturing & - & - & - & 84,660 & 88,826 & 104,327 \\
\hline \multicolumn{7}{|l|}{ Fabricated Metal Product } \\
\hline Manufacturing & - & - & 71,071 & 75,909 & 84,219 & 82,298 \\
\hline Public Administration & 51,765 & 60,731 & 69,436 & 79,484 & 86,497 & 84,927 \\
\hline \multicolumn{7}{|l|}{ Motor Vehicle } \\
\hline Manufacturing & 60,980 & 75,954 & 90,041 & 100,864 & 114,249 & 124,668 \\
\hline \multicolumn{7}{|l|}{ Professional, Scientific, and } \\
\hline Technical Services & 53,497 & 72,316 & 81,400 & 92,158 & 104,647 & 128,232 \\
\hline R\&D Organizations & - & 68,961 & 79,624 & 90,306 & 105,970 & 134,414 \\
\hline Utilities & 61,400 & 70,157 & 75,177 & 86,132 & 101,880 & 110,007 \\
\hline \multicolumn{7}{|l|}{ Electric Power Generation, } \\
\hline Transmission \& Distribution & - & 70,423 & 72,641 & 82,335 & 103,838 & 112,005 \\
\hline Mining & - & 74,627 & 84,008 & 95,928 & 111,883 & 123,899 \\
\hline All Nonmanufacturing & 52,245 & 65,267 & 73,742 & 83,016 & 90,437 & 99,471 \\
\hline All Manufacturing & 63,365 & 75,849 & 88,595 & 98,958 & 109,767 & 112,374 \\
\hline
\end{tabular}

